EPJ Web of Conferences 101, 04003 (2015)

DOI: $10.1051 /$ epjconf/ 201510104003

(C) Owned by the authors, published by EDP Sciences, 2015

\title{
Pulsations in close binaries: challenges and opportunities
}

\author{
C. Maceroni ${ }^{1, a}$, H. Lehmann ${ }^{2}$, R. Da Silva ${ }^{3}$, and J. Montalbán ${ }^{4}$
}

1 INAF - Osservatorio Astronomico di Roma, via Frascati 33, Monteporzio C., Italy

2 Thüringer Landessternwarte Tautenburg, Sternwarte 5, Tautenburg, Germany

3 Dipartimento di Fisica, Università di Roma Tor Vergata, via della Ricerca Scientifica 1, Rome, Italy

4 Dipartimento di Fisica e Astronomia, Università di Padova, vicolo dell'Osservatorio 3, Padova, Italy

\begin{abstract}
CoRoT and Kepler provided a precious by-product: a number of eclipsing binaries containing variable stars and, among these, non-radial pulsators. This providential occurrence allows combining independent information from two different phenomena whose synergy yields scientific results well beyond those from the single sources. In particular, the analysis of pulsations in eclipsing binary components throws light on the internal structure of the pulsating star, on the system evolution, and on the role of tidal forces in exciting the oscillations. The case study of the Kepler target KIC 3858884 is illustrative of the difficulties of analysis and of the achievements in this rapidly developing field.
\end{abstract}

\section{Introduction}

Eclipsing binaries (EB) with double-lined spectra allow to determine, in favorable cases, masses and radii of the components with an accuracy better than $1 \%$, by means of a purely geometrical method. Moreover, the binary components can be assumed to be coeval and of the same chemical composition. These constraints are extremely useful in the asteroseismic study of pulsating components of EBs, since they allow to discriminate among possible pulsation models and provide an independent check of the asterosismic determination of stellar parameters. A fast development of these studies was possible thanks to the light curves obtained by the space missions CoRoT and Kepler, which are characterized by unprecedented accuracy, continuous monitoring (from months to years) and high frequency sampling. Pulsation, however, adds complexity to data analysis, as the effects of the two phenomena shall be disentangled, or consistently modeled. An illustrative example of the achievements and difficulties met in this type of analysis is that of the Kepler target KIC 3858884, which we briefly present here. The complete study can be found in [4].

\section{A case study: KIC 3858884}

KIC 3858884 is a bright target $(V=9.28)$ which was almost continuously monitored for about 1100 days (Fig. 1). The light curve shows deep eclipses, complex pulsation patterns with pulsation frequencies typical of $\delta \mathrm{Sct}$, and a highly eccentric orbit $(e \simeq 0.5)$. Because of the high eccentricity, binarity affects also the out-of-eclipse sections of the light curve, in spite of the long orbital period $\left(P=25.95200^{\mathrm{d}}\right)$ and the small fractional radii $(<1 \%)$ of the components. Ground based spectroscopic follow-up provided the radial velocity (RV) curves displayed in Fig. 2.

The disentangling of pulsation and orbital effects is frequently achieved by an iterative method consisting in the alternate subtraction from the initial light curve of the harmonic and EB fits as, for instance, in [3]. The initial harmonic fit is usually computed from a light curve with masked eclipses.

\footnotetext{
a e-mail: maceroni@oa-roma.inaf.it
}

This is an Open Access article distributed under the terms of the Creative Commons Attribution License 4.0, which permits unrestricted use, distribution, and reproduction in any medium, provided the original work is properly cited. 

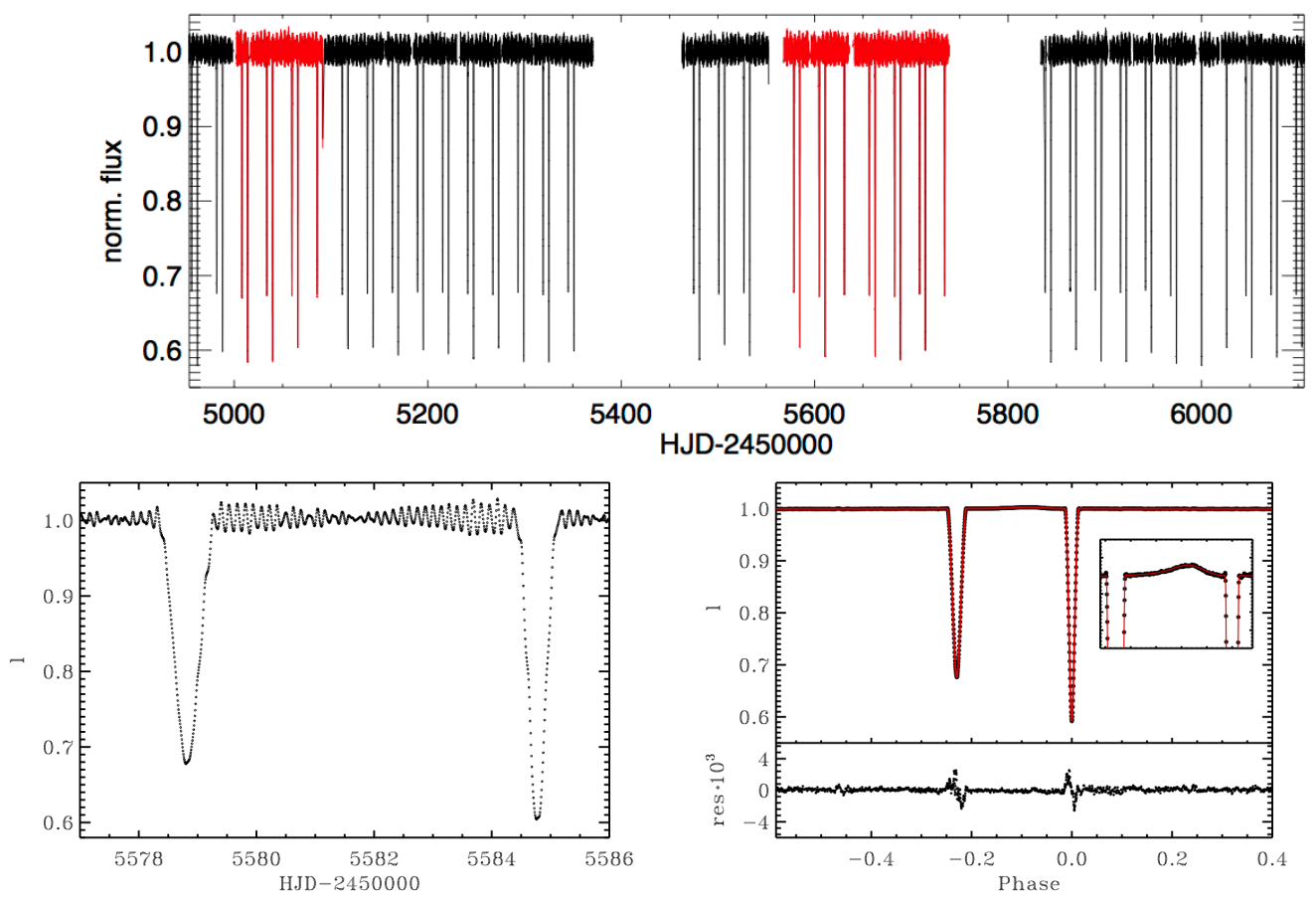

Fig. 1. Upper panel: Kepler light curve of KIC 3858884 after detrending and sigma-clipping removal of evident outliers. The sections in red correspond to short cadence sampling $\left(1^{\mathrm{m}}\right)$, the rest to long cadence $\left(29.4^{\mathrm{m}}\right)$. Lower left panel: a blow-up of the curve. Lower right: the disentangled binary light curve and (in red) the fit with PHOEBE; in the inset: blowup around periastron phase.

This method, however, is successful when one phenomenon affects the light curve much less than the other (e.g. grazing eclipses or pulsations of small amplitude with respect to the eclipse depth). This is not the case of KIC 3858884, since the subtraction of the same harmonic fit at all orbital phases, following the standard procedure, significantly increased the scatter around the secondary minimum. That indicates a partial visibility of the pulsator (identified thanks to this effect as the secondary component). We had therefore to take into account the decrease of its contribution to total light and change accordingly the pulsation amplitude during its eclipse.

The light curve, after pulsation removal, was computed with PHOEBE [6]. The best model was selected with a genetic algorithm searching for the global minimum of a cost function, $\chi^{2}$, computed from the residuals between the synthetic light curve and the observations (Fig. 1, lower right panel). PHOEBE was also used for the radial velocity curve fit, and the combined results provide the absolute system parameters in Table 1 .

Subtraction of the eclipsing binary light curve allowed a detailed harmonic analysis, yielding about 400 significant frequencies (Fig.3). In particular, a number of frequencies in the $\gamma$ Dor domain were detected, and the highest amplitude ones show the frequency ratio expected for high order g-modes of given spherical degree [5]. This suggested KIC 3858884 to be a hybrid $\gamma$ Dor/ $\delta$ Sct pulsator.

Further information on the physical properties of the system was derived by comparison with theoretical models. In particular, the evolution of the stellar radii with time, combined with the constraint of coevality, was used to infer the system age. The evolutionary tracks were obtained from stellar evolution modelling with the code CLES, Code Liégeois d'Évolution Stellaire [7], further details on the input physics can be found in [4]. Convection was treated by the mixing length theory, with the value of $\alpha_{\text {MLT }}$ fixed to 1.8. Models with and without overshooting were computed with the extra-mixing length expressed in terms of the local pressure scale height $\left(H_{\mathrm{p}}\right)$, as $\alpha_{\mathrm{OV}}$, and $\alpha_{\mathrm{OV}}$ values equal to 0.15 and 0.20 . A fine grid of models was computed with masses ranging between 1.82 and $1.91 \mathrm{M}_{\odot}$ and a 


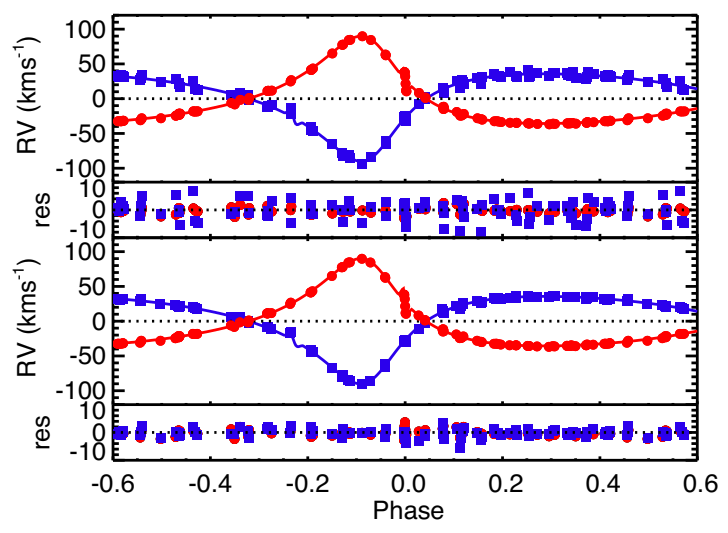

Fig. 2. Upper panel: the phased RV shifts of KIC 3858884 components as obtained from KOREL [2] and, below, the fit residuals. Filled red circles: primary star. Filled blue squares: secondary star. Curves: PHOEBE fit. Lower panel: the same after prewhitening with the two significant frequencies found in the residuals (the same highest amplitude ones found in the light curve)

Table 1. Orbital and physical parameters of KIC 3858884

\begin{tabular}{lc|lcc}
\hline & System & & Primary & Secondary \\
\hline$i\left(\left(^{\circ}\right)\right.$ & $88.176 \pm 0.002$ & $T_{\text {eff }}(\mathrm{K})$ & $6800^{a} \pm 70$ & $6606 \pm 70$ \\
$e$ & $0.465 \pm 0.002$ & $M\left(M_{\odot}\right)$ & $1.88 \pm 0.03$ & $1.86 \pm 0.04$ \\
$\omega$ & $21.61^{\circ} \pm 0.01$ & $R\left(R_{\odot}\right)$ & $3.45 \pm 0.01$ & $3.05 \pm 0.01$ \\
$q$ & $0.988 \pm 0.02$ & $\log g$ & $3.63 \pm 0.01$ & $3.74 \pm 0.01$ \\
$a\left(R_{\odot}\right)$ & $57.22 \pm 0.22$ & & & \\
\hline
\end{tabular}

a Value fixed by the spectroscopic analysis

step of $0.005 \mathrm{M}_{\odot}$. The chemical composition assumed different values of the metal mass fraction $(Z)$, from 0.007 to 0.018 , and two values of the helium mass fraction $Y=0.27$, and 0.28 . The range of excited frequencies for each model was computed with the non-adiabatic pulsation code MAD [1]. The constraint from the binary solution (masses, radii, primary $\mathrm{T}_{\text {eff }}$, temperature difference) yield a system age of $1.38-1.49 \mathrm{~Gy}$, see Fig. 4. This age interval defines the consistent values of the minimum and maximum excited frequencies, both in the $\gamma$ Dor and $\delta$ Sct domain (in excellent agreement with the results of the harmonic analysis) and the values of the integral of the the Brunt-Väisälä frequency of the secondary star.

In conclusion the pulsation properties derived from the models and the binary constraints are in full agreement with the observations.

\section{References}

1. Dupret, M.-A., De Ridder, J., De Cat, P., et al., A\&A 398, (2003) 677

2. Hadrava, P. ASPC 318, (2004) 86

3. Maceroni, C., Montalbán, J., Gandolfi, D., Pavlovski, K., \& Rainer, M., A\&A 552, (2013) A60

4. Maceroni, C., Lehmann, H., da Silva, R., et al. A\&A 563, (2014) A59

5. Moya A., Suárez J. C., Amado P. J., Martin-Ruíz S., Garrido R., A\&A 432, (2005), 189

6. Prša, A., \& Zwitter, T. ApJ, 628, (2005) 426

7. Scuflaire, R., Théado, S., Montalbán, J., et al., Ap\&SS 316, (2008), 83 


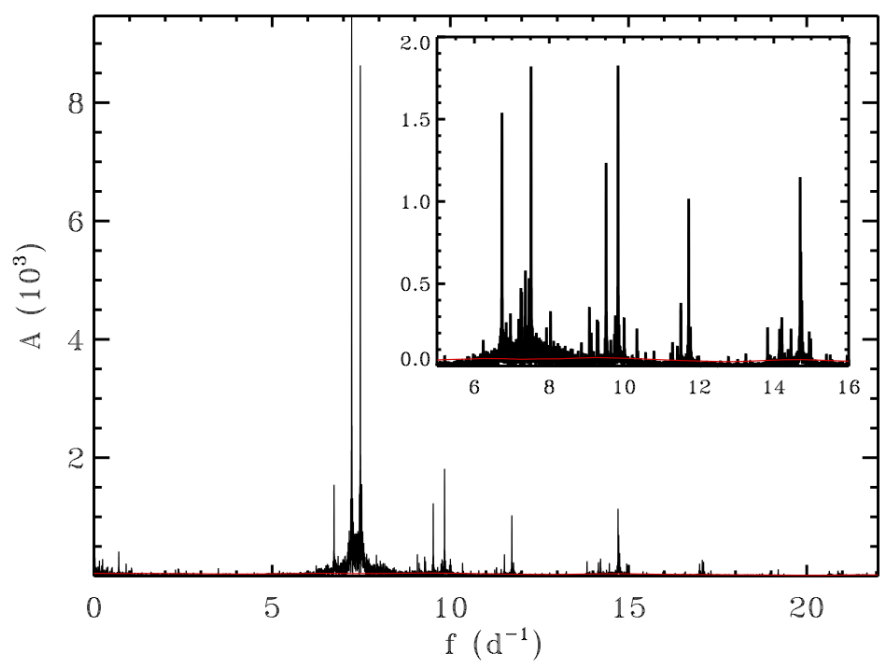

Fig. 3. The final amplitude spectrum of KIC 3858884. In the inset a blowup after prewhitening the two highest frequencies $\left(f_{1}=7.2306 \mathrm{~d}-1\right.$ and $\left.f_{2}=7.4374 \mathrm{~d}-1\right)$. The red line is a significance threshold as defined in [4].
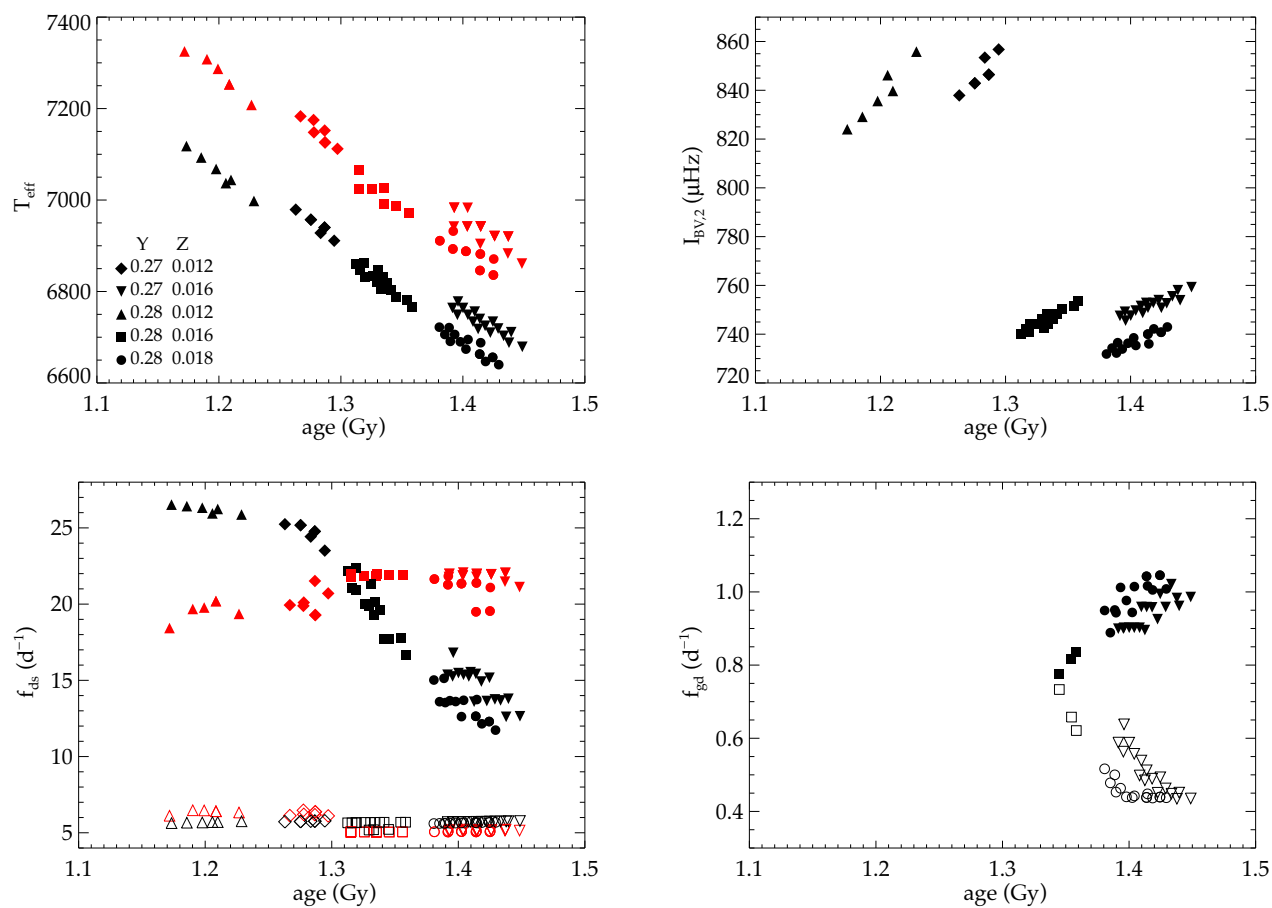

Fig. 4. The properties of the stellar models selected as explained in the text. In all panels different quantities are plotted as function of the model age, the symbol shape depends on the chemical composition, the red symbols correspond to the primary star, the black ones to the secondary. Top left panel: $T_{\text {eff }}$ for both stars; top-right panel: the integral of the Brunt-Väisälä frequency for the secondary component; bottom left panel: the minimum and maximum excited frequency in the $\delta$ Sct domain for both stars (empty and full symbols denote, respectively, the minimum and maximum values), bottom right panel: the minimum and maximum excited frequency in the $\gamma$ Dor domain for the secondary. 